\title{
Effect of Surface Sampling and Recovery of Viruses and Non- Spore-Forming Bacteria on a Quantitative Microbial Risk Assessment Model for Fomites
}

\author{
Mark H. Weir ${ }^{1,}$, Tomoyuki Shibata $^{2}$, Yoshifumi Masago ${ }^{3}$, Dena L. Cologgi ${ }^{4}$, Joan B. Rose ${ }^{5}$ \\ 1 -Departments of Epidemiology and Biostatistics and Civil and Environmental Engineering, \\ Temple University, 1301 Cecil B. Moore Ave. Ritter Annex 9 9h $^{\text {th }}$ Floor, Philadelphia, PA 19122 \\ ${ }^{2-}$ School of Nursing and Health Studies, Northern Illinois University \\ 3-Department of Civil and Environmental Engineering, Tohoku University \\ 4 -Department of Microbiology and Molecular Genetics, Michigan State University \\ 5 -Department of Fisheries and Wildlife, Michigan State University
}

\begin{abstract}
QMRA is a powerful decision analytics tool, yet it faces challenges when modeling health risks for the indoor environment. One limitation is uncertainty in fomite recovery for evaluating the efficiency of decontamination. Addressing this data gap has become more important due to response and recovery from a potential malicious pathogen release. In order to develop more accurate QMRA models, recovery efficiency from non-porous fomites (aluminum, ceramic, glass, plastic, steel and wood laminate) was investigated. Fomite material, surface-area (10, 100 and 900 $\mathrm{cm}^{2}$ ), recovery tool (swabs and wipes), initial concentration on the fomites and eluent (Polysorbate-80, trypticase soy broth and beef extract) were evaluated in this research. Recovery was shown to be optimized using Polysorbate-80, sampling with wipes and sampling a surfacearea of $10-100 \mathrm{~cm}^{2}$. The QMRA model demonstrated through a relative risk comparison, the need for recovery efficiency to be used in these models to prevent underestimated risks.
\end{abstract}

\section{Keywords}

Stochastic modeling; QMRA; fomites; recovery efficiency; site assessment; site characterization

\section{Introduction}

Fomites, QMRA and Infectious Disease

Quantitative microbial risk assessment (QMRA) is a modeling framework that informs vital decisions in re-occupancy evaluation [1], [2]. QMRA can be used to optimize site assessment and decontamination efficacy testing, fields heavily dominated by the use of surface sampling [3], [4]. QMRA will never replace surface sampling, but can improve the targeting

\footnotetext{
*- weirmarkh@gmail.com, Phone: 001-215-204-9160, Fax: 001-215-204-1854.
} 
and planning of such efforts and it can also be leveraged to determine the proper number of samples to optimize site characterization.

One of the greatest challenges to a QMRA modeler addressing the indoor environment is the ubiquity and consistent interaction between people and fomites ${ }^{[5]-[8]}$. Fomites are a dynamic exposure media that are contaminated typically by the room occupants themselves, via ejection of infectious particles and hand touching. Indoor populations are all but guaranteed to interact with fomites, therefore a robust method of including fomite analysis for site assessment and re-occupancy evaluation is essential [9], [10].

\section{Noted Effects by Sampling and Fomite Variables}

Since the Anthrax attacks in 2001 there has been extensive work on surface sampling for Bacillus anthracis (B. anthracis) spores ${ }^{[11]-[14]}$. However similar surface sampling research for non-spore forming bacteria and viruses remains limited as compared to $B$. anthracis [3], [15]-[17]. Indoor environmental conditions and two eluents' effect on recovery efficiency $\left(\eta_{R}\right)$ for plastic laminate and galvanized steel were quantified by previous researchers via a thorough literature review and their own experiments ${ }^{[18]}$. The $\eta_{R}$ results from ${ }^{[18]}$ showed microbial survival had an effect on $\eta_{R}$ and reinforced previous results of the effects of sampled surface-area ${ }^{[19]}$. Three primary areas of uncertainty have been highlighted for research into their impacts on $\eta_{R}$ and its use in QMRA, namely fomite type, fomite surfacearea and sampling material.

Fomite Type and Surface-area-Non-porous fomites have a higher microbial transfer efficiency (MTE) from fomite-to-hand, with a median MTE of $40 \%$ as opposed to porous fomites, with a median MTE of 5\% ${ }^{[4]}$. This may be due to porous fomites' characteristically increased roughness which decreases $\eta_{R}$ or due to the pores harboring pathogens [20]. Previous research into non-porous fomites demonstrated the difference in $\eta_{R}$ using sets of surface-areas for: aluminum, glass, plastic, wood laminate and galvanized and stainless steels [12],[18],[21]. In previous research it was shown that $100 \mathrm{~cm}^{2}$ provided optimal recovery for $B$. anthracis ${ }^{[19]}$, but this does not hold true in all cases, and may be different for nonspore forming bacteria and viruses.

Initial Concentration, Sampling Material-Sampling materials refer to both the recovery tool, typically swabs or wipes and eluents that are used to develop a suspension from the microorganisms on the recovery tool. Isolating $\eta_{R}$ effects for realistic candidate sampling materials will inform optimal sampling materials for this research and site characterization. Of the recovery tools, wet macrofoam swabs have been shown to be effective, obtaining consistently high microbial recoveries ${ }^{[11], ~[12]}$. Due in part to their relatively large surface-areas, wipes are considered an effective surface sampling tool, especially for larger fomite surface-areas ${ }^{[10]}$. Sponge materials can theoretically achieve similar recovery levels as swabs, however, this similarity does not continue for microbes [21],[14],[22]. Therefore for their demonstrated capabilities in microbial surface sampling, swabs and wipes are used in this research.

Of the eluents considered, polysorbates (brand name Tween) are often used. In previous research Polysorbates have been compared to trypticase soy broth (TSB) and water ${ }^{[18]}$ with 
TSB demonstrating better $\eta_{R}$ potential. This research investigates this potential $\eta_{R}$ improvement between PS80 and TSB and assesses the effectiveness of a neglected option, beef extract.

Scope of Research-This research is targeted to address key knowledge gaps in $\eta_{R}$ and the development of QMRA models for fomites. Therefore this research develops an example QMRA model for fomites. Site assessment like all microbial sampling is typically an expensive task. Therefore a re-occupancy QMRA model derived around a hypothetical indoor space was developed. This QMRA model is intended to demonstrate how to utilize $\eta_{R}$ to assist in targeting which fomite(s) should be sampled more frequently to maintain health protection without over-sampling fomites associate with a lower health risk probability.

\section{Methods and Materials}

\section{Experimental Methods}

Inoculum Development-Enterobacteria phage P22 (P22) was used as the pathogenic virus surrogate ${ }^{1}$ with Salmonella enterica ( $S$. enterica ${ }^{2}$ ) as the bacterial host. Using $25 \mathrm{ml}$ of $\mathrm{TSB}^{3}$ S. enterica was grown to $\log$ phase for $0.1 \mathrm{ml}$ of P22 stock. After incubating for 24 hours at $37^{\circ} \mathrm{C}$, a solution for centrifugation (2,390 $\mathrm{g}$ for 10 minutes) was developed using $0.1 \mathrm{ml}$ of lysozyme $(50 \mathrm{mg} / \mathrm{ml})$ and $0.75 \mathrm{ml}$ of $0.5 \mathrm{M}$ EDTA. The resulting $25 \mathrm{ml}$ of solution was filtered at $0.22 \mu \mathrm{m}$ to remove bacteria particles.

Staphylococcus aureus ( $S$. aureus ${ }^{4}$ ) was used as the surrogate for pathogenic non-spore forming bacteria. $S$. aureus was grown in $25 \mathrm{ml}$ of TSB incubated at $35^{\circ} \mathrm{C}\left( \pm 2^{\circ} \mathrm{C}\right)$ for 24 hours.

Sampling Material Control Experiments-To assess an optimal recovery method two control experiments were conducted for: BE, PS80 and TSB as eluents and both swabs and wipes as sampling tools. This was accomplished via two control experiments. First to determine if there are limitations to recovery or growth on the sampling tool, swabs ${ }^{5}$ and wipes ${ }^{6}$ were directly inoculated. The swabs were dipped into phosphate buffered saline with $0.01 \%$ polysorbate- 80 and excess liquid was expressed by pressing the swab against the inside test-tube wall. Direct inoculation trials on the swabs and wipes started with, $10^{4} \mathrm{CFU}$ in a total volume of $10 \mu \mathrm{l}$, added directly to the swabs and wipes. Second to evaluate the optimal sampling tool, a dose of $1.5 \cdot 10^{3} \mathrm{PFU}$ was added to $10 \mathrm{~cm}^{2}$ ceramic (CR) fomite and allowed to dry in a safety cabinet at $25^{\circ} \mathrm{C}\left( \pm 2^{\circ} \mathrm{C}\right)$ and $50 \%$ relative humidity $(20$ minute drying time). The fomite sampling protocol outlined in section 2.1.4 of this manuscript was used to sample the $\mathrm{CR}$ fomite for this control experiment. As a validation this second control experiment was replicated on plastic (PL) fomite samples.

${ }_{1}^{1}$ Obtained from Charles P. Gerba of the University of Arizona

${ }_{3}^{2}$ Typhimurium strain LT2 ATTC\#19585

3\#211768, Becton Dickinson, Franklin Lakes, NJ

${ }_{5}^{4}$ ATTC: 25923

5 VWR Critical Swab, 10812-006, Suwannee, GA

${ }^{6}$ Cat\# FEL99715, STRATUS Inc., Amarillo, TX 
Effects to $\eta_{R}$ from initial fomite inoculation concentration was quantified by inoculating all six fomite materials with $10^{2}$ and $10^{7} \mathrm{PFU} \cdot \mathrm{cm}^{-2}$ of P22 and $10^{3}$ and $10^{6} \mathrm{CFU} \cdot \mathrm{cm}^{-2}$ of $S$. aureus. The fomite sampling protocol outlined in section 2.1.4 of this manuscript was used for this control experiment.

Fomite Inoculation for Surface-area Experiments-Six different non-porous fomites were tested: aluminum (AL), ceramic (CR), glass (GL), plastic (PL), stainless steel (ST) and wood laminate (WL). For each fomite three surface-areas were evaluated: 10, 100 and 900 $\mathrm{cm}^{2}$. Fomite surfaces were inoculated with $10-20 \mu \mathrm{L}$ of the P22 and $S$. aureus solutions independently using single channel pipettes. Inoculation was carried out using $10^{3}$ to $10^{7}$ PFU and CFU cm ${ }^{-2}$ for P22 and $S$. aureus respectively. Three fomite samples for each fomite type and surface-area were prepared (cleaned and sterilized) and inoculated with each initial concentration. After inoculation each of the fomites were stored in a safety cabinet at an average temperature of $25^{\circ} \mathrm{C}\left( \pm 2^{\circ} \mathrm{C}\right)$ and relative humidity of $50 \%$ until the surfaces were completely dry (approximately 20 minutes). Each inoculation trial was run in triplicate resulting in 54 sampling results for analysis and modeling.

Fomite Sampling and Sample Assays-Each of the fomite test samples was wiped horizontally, vertically then diagonally five times in each direction. Swabs were periodically rolled to expose unused swab surfaces. Wipes had a surface-area of $12 \mathrm{~cm}^{2}$ and were periodically folded to expose unused wipe surfaces. To eliminate effects of growth from nutrients in the eluents, fomite experiments were organized to eliminate hold time between sampling, elution and assay.

Once the fomite surfaces were sampled each swab and wipe was placed into $50 \mathrm{ml}$ tubes each containing $10 \mathrm{ml}$ of eluent: BE, PS80 and TSB. The tubes were vortexed for 30 seconds to elute the samples. P22 was assayed using EPA Method 1602 [23] with a slight modification: $1 \mathrm{ml}$ of the extracted samples added to $10 \mathrm{ml}$ tubes containing $3 \mathrm{ml}$ of TSB and $1 \%$ melted agar ${ }^{7}$ and maintained at $49^{\circ} \mathrm{C}$ with $300 \mu$ of the $S$. enterica in log phase. The tubes were hand mixed and poured onto trypticase soy agar plates, after solidification incubated at $37^{\circ} \mathrm{C}$ for 24 hours. Resulting plaques on the plates were counted as P22.

Direct spreading was used for $S$. aureus where: $100 \mu \mathrm{l}$ of extracted sample was pipetted onto BBL Mannitol Salt Agar plates 8 and drops were spread across the surface using a sterile glass rod. The plates were incubated aerobically at $35^{\circ} \mathrm{C}$ for 48 hours. Yellow-zoned colonies were counted as $S$. aureus. All of the microbiological assays were performed in triplicate.

\section{Modeling Methods}

Exposure Model-The QMRA model simulates the contamination and subsequent risks to users of a shared office or workspace. In the office three generic users have worked individually and consecutively over one day, with a two-hour exposure time for each person. The QMRA model simulates the relative risks to a user based on fomite exposure after re-

${ }^{7}$ Bacto agar \#214010, Becton Dickinson, Sparks, MD

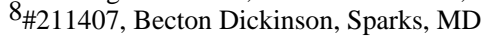


occupancy. The model simulates the first users' contamination of office fomites via hand contact. This simulated contamination event was used to mimic discrete direct contamination from an infected individual contaminating a shared workspace. This hypothetical scenario was developed to simulate a shared workspace such as a computer laboratory or rented teleconference rooms (as limited examples). Thus allowing for a realistic yet limited scenario developed to demonstrate the use of $\eta_{R}$ in a QMRA model for fomites.

A discrete time Markov chain (MC) model was used to model: the contamination of fomites, concentration on fomites over time and the exposure of subsequent users to the contaminated fomites. Three typical office fomites were modeled: WL simulating a desk surface, PL simulating computer surfaces (monitor edge, and peripherals) and AL simulating a filing cabinet, desk drawers and doorknob. Figure 1 shows a conceptual diagram of the exposure model that consists of three coupled independent MC models.

The exposure model is built so that with each fomite contact there is a likelihood of transfer to both the hand and the fomite. The first user contaminates the fomites (AL, PL and WL) over the course of 2 hours of use. Then, two subsequent users are exposed when using the office for 2 hours each ( 0.5 hour lag period between users). The output from each of the MC models used in risk modeling is the concentration of pathogens remaining on the fomites (e.g. available for sampling).

Markov Model Construction-Markov processes use transition probabilities to estimate the likelihood of pathogens being transported from one state ${ }^{9}$ to another. Loss rates derived from fundamental physical, biochemical or microbiological process(es) are used to develop the transition probability values [24], [25].

Within a given time step $(\Delta t) p_{i i}$ is the probability of being in state- $i$ and remaining in state- $i$. By taking the sum of all losses in state- $i\left(\lambda_{i}\right)$ we can develop an exponential survival probability to describe $p_{i i}$ (equation 1 ). An unconditional probability can be used to model pathogen transport from state- $i$ to state- $j\left(p_{i j}\right.$; equation 2$)$. A loss rate specific to transitioning from state- $i$ to state- $j\left(\lambda_{i j}\right)$ is used to construct the ratio of $\lambda_{i j}$ to $\lambda_{i}$, modeling the transport of pathogens from state- $i$. This ratio is multiplied by the compliment of $p_{i i}$ to account for the likelihood of remaining in state- $i^{[26]}$.

$$
\begin{aligned}
& p_{i i}=\exp \left(-\lambda_{i} \cdot \Delta t\right) \\
& p_{i j}=\frac{\lambda_{i j}}{\lambda_{i}} \cdot\left[1-p_{i i}\right]
\end{aligned}
$$

Following the state list in Figure 1; the losses from user 1's hands to fomites are described in equation 3 , where the loss rate is dependent on:

- $\quad$ Finger-tip per touch surface-area $\left(A_{p t}, 0.0108 \mathrm{ft}^{2}\right)$ for 10 fingers ${ }^{[13]}$

\footnotetext{
${ }^{9}$ Physical location for pathogens (i.e. human hands and fomites)
} 
- $\quad$ surface-area of the fomite $\left(A_{f}\right)$ value varies depending on the fomite

$$
\text { - } \quad A_{A L}=0.017 \mathrm{ft}^{2} ; A_{P L}=0.638 \mathrm{ft}^{2} ; A_{W L}=11.25 \mathrm{ft}^{2}
$$

- $\quad$ transfer efficiency from hand-to-fomite, assumed to be constant $\left(\eta_{t-h f}, 0.715\right.$ unit-less) [4]

- $\quad$ and frequency of touching fomites assumed constant for all fomites $\left(\lambda_{i} ; 0.2\right.$ min $-1)[13]$

The losses from fomites-to-hands are described in equation 4, which is similar to equation 3 except in that $\eta_{t-h f}$ is replaced with the transfer efficiency from fomites-to-hands $\left(\eta_{t-f h}\right.$; 0.409 unit-less) ${ }^{[4]}$. Inactivation was considered constant at $0.0016 \mathrm{~min}^{-1}$ for all states [13], [26]. All exposure model parameters and values are in supplementary information.

$$
\begin{aligned}
& \lambda_{a 12}, \lambda_{a 13}, \lambda_{a 14}
\end{aligned}
$$

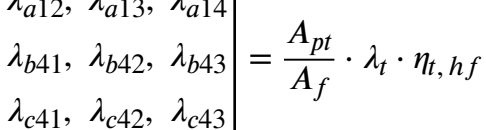

$$
\begin{aligned}
& \lambda_{a 21}, \lambda_{a 31}, \lambda_{a 41}
\end{aligned}
$$

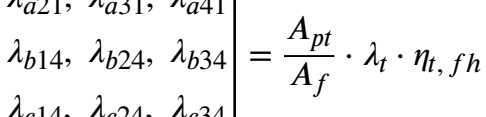

Before the Markov chain model was executed, equation 5 was used to estimate the load of microorganisms on the fomite $\left(N_{f}\right.$, CFU or PFU) dependent on: surface-area of the fomite $\left(A_{f}\right)$ and a hypothetical initial concentration inoculated $\left(C_{i}, 100,000 \mathrm{CFU}\right.$ or PFU $\left.\cdot \mathrm{cm}^{-2}\right)$. Since none of the assayed fomite samples demonstrated growth a result that is supported through previous non-porous fomite research ${ }^{[27]}$ growth is not considered in this QMRA model.

$$
N_{f}=C_{i} \cdot A_{f}
$$

Infection Risk Modeling-Since this is a hypothetical risk scenario, the pathogen simulated is infectious $S$. aureus, chosen for its ability to develop cutaneous and subcutaneous infections. The exponential dose response model (equation 6) with a $k$ of $7.63\left(10^{-8}\right)$ was chosen to model the risk $\left(R_{i}\right)^{[28]}$. The simulated dose (number of microorganisms) remaining on fomites was used to estimate the relative risks posed by individual fomites and all three fomites combined.

$$
R_{i}=1-\exp (-k \cdot d o s e)
$$

Since the actual risks to occupants are a combination of all fomites, the risks from each fomite should be coupled. Equation 7 is an expansion of the inclusion exclusion principle that models the combined risks posed from the fomites in the room [29]. This was only 
performed for two occupants but demonstrates the simple generalizable form of the QMRA model.

$$
P\left(R_{E}\right)=\left|\bigcup_{i=1}^{n} R_{i}\right|=\sum_{j=1}^{n}(-1)^{j+1}\left(\sum_{1 \leq i_{1} \leq \cdots \leq i_{j} \leq n}\left|R_{i} \cap \ldots \cap R_{i j}\right|\right)
$$

\section{Code Verification}

The Markov model and all analyses performed were written and executed in the $\mathbf{R}$ computational programming language (http://www.r-project.org/). Therefore the code was verified by operating the model for a small number of iterations with the calculated pathogen concentration in each state being concurrently calculated by hand to check their values were correct.

\section{Results}

\section{Recovery Method Comparison and Optimal Method Determination}

By conducting the fomite recovery assays in triplicate, 9 data points for each of the fomite surface-area sets were developed. A chi-squared power analysis showed a low effect due to this sample size $(\mathrm{w}=0.045)^{[30]}$. The Kruskall-Wallace test $(a=0.05)$ was used to compare the experimental parameters. $\eta_{R}$ was calculated using equation 8 where: $M_{a}$ is the number of colonies on the agar plate (CFU or PFU), $M_{i}$ is the number of colonies inoculated on the fomite and $D$ is the dilution-factor: volume of reagent for elution from sampling tool divided by volume of sample assayed.

$$
\eta_{R}=\frac{M_{a} \cdot D}{M_{i}}
$$

Sampling Material Control Experiments-When directly inoculated with $10^{4} \mathrm{PFU}$ of P22, swabs provided better $\eta_{R}$ than wipes when eluted with TSB, BE and PS80, resulting in 136, 110 and $114 \% \eta_{R}$ respectively. Using TSB, BE and PS80 wipes allowed for 106, 94 and $104 \% \eta_{R}$ respectively (table 1 ). This average performance difference was not statistically significant ( $p$-value $=0.087$ ). These results suggest that potential microorganism loss during elution were negligible, but rather it suggests that growth was associated with these materials. However these growth results do not continue for fomites, thus the growth is only observed for directly-inoculated sampling tools.

Sampling Material and Eluent Comparison-Table 1 shows that when a dose of $1.5 \cdot 10^{3} \mathrm{PFU}$ was added to a $10 \mathrm{~cm}^{2} \mathrm{CR}$ fomite, wipes resulted in a median $\eta_{R}$ of $69 \%$ (range of $60-70 \%$ ), swabs only achieved a median $\eta_{R}$ of $10 \%$ (range of $7.8-15 \%$ ). This was confirmed using a PL fomite with the same inoculation dose $\left(1.5 \cdot 10^{3} \mathrm{PFU}\right)$ Wipes resulted in a median $\eta_{R}$ of $56 \%$ (range of $28-79 \%$ ) and swabs resulted in a median $\eta_{R}$ of 
$13 \%$ (range of $3-22 \%$ ) from the PL fomite. The $\eta_{R}$ differences between wipes and swabs were statistically significant for both CR and PL fomites ( $p$-value $=0.00085)$.

Using wipes the optimal eluent was determined. When using TSB, BE and PS80 $\eta_{R}$ were 64, 48 and 74\% respectively (averaged between both $S$. aureus and P22). While not statistically significant, PS80 demonstrated higher $\eta_{R}$ when compared to other eluents. Based on the results from these two controls the combination of wipes and PS80 were used for the experiments to quantify the effects of initial concentration, fomite material and fomite surface-area on $\eta_{R}$

\section{Fomite and Exposure Factors Effect on Recovery}

Effects to $\eta_{\boldsymbol{R}}$ from Initial Concentration on Fomite-Initial concentration control experiment results can be seen in table 2. An initial concentration of $10^{2} \mathrm{PFU} \cdot \mathrm{cm}^{-2}$ showed a mean $\eta_{R}$ for all fomites of $73 \%$ (range of $68-78 \%$ ). For $10^{7} \mathrm{PFU} \cdot \mathrm{cm}^{-2}$ a mean $\eta_{\mathrm{R}}$ for all fomites of $62 \%$ (range of $53-68 \%$ ) was achieved. This greater $\eta_{R}$ for $10^{2} \mathrm{PFU} \cdot \mathrm{cm}^{-2}$ was statistically significant $(p$-value $=0.0013$ ) for P22.

For $S$. aureus an initial concentration of $10^{3} \mathrm{CFU} \cdot \mathrm{cm}^{-2}$ resulted in a mean $\eta_{R}$ for all fomites of $77 \%$ (range of $68-88 \%$ ). For $10^{6} \mathrm{CFU} \cdot \mathrm{cm}^{-2}$ mean $\eta_{R}$ for all fomites was $82 \%$ (range of $65-92 \%$ ). Differences in $\eta_{R}$ for $S$. aureus were not statistically significant for initial concentration of microorganisms.

Fomite Surface-area-Effects of fomite surface-area on $\eta_{R}$ are summarized in Table 3 and Figure 2. P22 on $10 \mathrm{~cm}^{2}$ fomite attained a mean $\eta_{R}$ for all fomites of $73 \%$ (range of 68 $-78 \%$ ) and for $S$. aureus a mean $\eta_{R}$ for all fomites of $77 \%$ (range of $68-88 \%$ ). For 100 $\mathrm{cm}^{2}$ surface-area P22 reached a mean $\eta_{R}$ for all fomites of $49 \%$ (range of $41-55 \%$ ) and for $S$. aureus a mean $\eta_{R}$ for all fomites of $21 \%$ (range of $10-30 \%$ ). For $900 \mathrm{~cm}^{2}$ surface-area P22 resulted in a mean $\eta_{R}$ for all fomites of $25 \%$ (range of $10-43 \%$ ) and for $S$. aureus a mean $\eta_{R}$ for all fomites of $10 \%$ (range of $6.6-17 \%$ ). All surface areas are statistically significantly different from each other.

\section{Fomite QMRA}

Figure 3 shows the resulting risk estimates for each of the fomites and the combined estimated risk values. As can be seen in figure 3 the second user of the office invariably has a lower risk of infection associated with the fomites. This is likely due to the survival of the pathogens combined with the relatively low transmission rates. Therefore as a general example a site assessor may conclude that there is the potential for natural attenuation to control the contamination in this office, but would want to simulate longer time periods than this example simulation. Also shown in figure 3, despite AL being the fomite with the lowest surface-area in the hypothetical office, AL contributed the most to the combined risks $(E[R s])$. WL fomites demonstrated the lowest risks posed to the office users, with PL fomites the second highest.

Figure 3 shows the effect fomite $\eta_{R}$ has on the resulting $E[R s]$. A consistent trend of underestimated risks from not considering $\eta_{R}$ can be seen. This means that decisions based on these underestimated risks are not as protective as thought. A differential method for the 
sensitivity analysis showed that $\eta_{R}$ was not the greatest contributor to model variance (figure 4). Rather the known or assumed loading to surfaces was the model variable producing the greatest variance. When fomite $\eta_{R}$ is included then this is the second highest contributor to model variance When recovery is not considered, surface-area of the fomite is the second greatest cause of model variance. When $\eta_{R}$ is not included, there is no means of assessing the sensitivity of this variable in the model, thus no black bar for this portion of the sensitivity plot.

\section{Discussion}

Experimental results demonstrated that using wipes with PS80 as an eluent provided for optimal $\eta_{R}$ Through experimentation it was also reinforced from previous research ${ }^{[19]}$ that focusing on a sampling surface-area of $10-100 \mathrm{~cm}^{2}$ provides for greatest $\eta_{R}$ This research also reinforces the conclusion that wipes are an optimal sampling tool for site characterization [11], [12]. Therefore it is recommended to use the $10-100 \mathrm{~cm}^{2} \eta_{R}$ for site assessors and QMRA modelers. Use of $\eta_{R}$ only from this research would limit the sampling protocol to sampling surface-areas consistent with those for which these $\eta_{R}$ were developed. Therefore based on this limitation and other varying indoor space conditions it is recommended that multiple recommended $\eta_{R}$ values and expert guidance are considered by site assessors for those fomites being sampled and modeled.

The potential growth on the directly inoculated sampling tools could have had significant effects on the assessment of recovery efficiency had this result continued into the fomite experiments. There was no hold time between fomite sampling, elution and subsequent assay resulting in no observed growth during the fomite experiments. Therefore it can be concluded that this growth on the sampling tool is negligible to the $\eta_{R}$ from fomites, reinforcing results from ${ }^{[27]}$. Research is currently underway to investigate a means of computationally accounting for this growth potential in a sampling protocol QMRA, which is a fundamentally different model from a fomite QMRA. Further experimental research is also recommended to conclusively resolve this question of the effect of growth on the sampling tools.

This QMRA model demonstrates that after an incidental contamination of fomites from one user of an office, there is a degrading risk of infection to the subsequent user(s). Understanding how the contamination is changing due to natural processes allow for inferences regarding re occupancy and decontamination. Since the same frequency of touching was used in the exposure model, the effect of fomite material was isolated. An important inference that can be made from a fomite QMRA model is targeted sampling and analysis of fomites identified to provide a higher risk potential. This was partially demonstrated with the AL fomites' greater impact on the coupled risks, despite being the smallest fomite surface-area in the room. An interesting expansion of this would be to develop a QMRA model where touch frequencies specific to the fomite material are used. This would not be as generalizable but expand on the relative risks from each of the fomites and be more realistic. A site assessment team can infer from a similar QMRA model developed for their scenario and room(s) that, AL fomites should be sampled more after decontamination to ensure re-occupancy safety. 
Initial pathogen loading to the fomites was the parameter causing the QMRA model the most variance; therefore establishing operating procedures for optimal recovery is vital. QMRA model sensitivity indicates fomite surface-area as the second most sensitive factor, corroborated by its significant experimental effect on $\eta_{R}$ The recovery while not driving the sensitivity of the QMRA model has a significant effect on it. Given the importance of site cleanup and remediation a trend of underestimated risks due to not accounting for $\eta_{R}$ would result in ineffective control measures.

The inclusion of additional room and fomite parameters will strengthen future QMRA models developed for similar scenarios. However the structure of this fomite QMRA model is the important step forward. QMRA models are all scenario dependent but typically share a basic mathematical structure. This model demonstrates a generalizable mathematical structure for using recovery efficiencies in the QMRA model to assess indoor sampling requirements. Additional factors such as response time of the sampling and site assessment personnel are those that would expand the scenario to be specific for the user. This is not unlike other QMRA models where the framework and basic mathematical methods are the same or very similar, but the model must be adapted to the users' scenarios and needs [31], [24]. This is due to the scenario dependency of QMRA models [1].

For situations such as establishing water sanitation and health (WASH) protocols and procedures, this type of modeling is very valuable. An improved QMRA model will allow for WASH planning to be more accurate, especially in the light of highly persistent, virulent or transmissible pathogens. The virus and bacteria surrogates are generalizable to other pathogens since the hold times were not long enough to encounter death rates of the microorganisms.

\section{Supplementary Material}

Refer to Web version on PubMed Central for supplementary material.

\section{Acknowledgements}

Funding for this research was supported by Center for Advancing Microbial Risk Assessment (CAMRA) STAR grant \#R83236201 and QRMA III supported by NIGMS/NIH, Award Number R25GM108593. We would like to acknowledge Dr. Sylvia Twersky of Temple College of Public Health for her assistance in the table of contents art.

\section{References}

[1]. Haas CN, Rose JB, and Gerba CP, Quantitative Microbial Risk Assessment, Second J. Wiley and Sons, 2014.

[2]. Huang Y, Hong T, Bartrand TA, Gurian PL, Haas CN, Liu R, and Tamrakar SB, "How Sensitive Is Safe? Risk-Based Targets for Ambient Monitoring of Pathogens," IEEE Sens. J, vol. 10, no. 3, pp. 668-673, Mar. 2010.

[3]. Nelson J, Shinn AM, and Bivens A, “Operating Room Telephone Microbial Flora,” 52005.

[4]. Rusin P, Maxwell S, and Gerba CP, "Comparative Surface to Hand and Finger to Mouth Transfer Efficiency of Gram-Positive, Gram-Negative Bacteria and Phage,” J. Appl. Microbiol, vol. 93, pp. 585-592, 2002. [PubMed: 12234341]

[5]. Roundy RW, "Human behaviour and disease hazards: Spatial perspectives on rural health," GeoJournal, vol. 3, no. 6, pp. 579-586, Nov. 1979. 
[6]. Hall CB, Douglas RGJ, and Geiman JM, "Possible Transmission by Fomites of Respiratory Syncytial Virus.,” J. Infect. Dis, vol. 141, pp. 98-102, 1980. [PubMed: 7365274]

[7]. Julian TR, Canales RA, Leckie JO, and Boehm AB, “A Model of Exposure to Rotavirus from Nondietary Ingestion Iterated by Simulated Intermittent Contacts,” Risk Anal, vol. 29, no. 5, pp. 617-632, 5 2009. [PubMed: 19187484]

[8]. Li S, Eisenberg JNS, Spicknall IH, and Koopman JS, "Dynamics and Control of Infections Transmitted From Person to Person Through the Environment," Am. J. Epidemiol, vol. 170, no. 2, pp. 257-265, Jul. 2009. [PubMed: 19474071]

[9]. Boone SA and Gerba CP, "The Occurrence of Influenza A Virus on Household and Day Care Center Fomites,” J. Infect, vol. 51, pp. 103-109, 2005. [PubMed: 16038759]

[10]. Reynolds KA, Watts P, Boone SA, and Gerba CP, "Occurrence of Bacteria and Biochemical on Public Surfaces," 15, pp. 225-234, 2005.

[11]. Rose L, Jensen B, Peterson A, Baner AN, and Arduino M, "Swab Materials and Bacillus anthracis Spore Recovery From Non-Porous Surfaces,” Emerg. Infect. Dis, vol. 10, pp. 10231029, 2004. [PubMed: 15207053]

[12]. Hodges LR, Rose LJ, Peterson A, Noble-Wang J, and Arduino MJ, "Evaluation of a Macrofoam Swab Protocol for the Recovery of Bacillus anthracis Spores from a Steel Surface," Appl. Environ. Microbiol, vol. 72, no. 6, pp. 4429-4430, 2006. [PubMed: 16751562]

[13]. Nicas M and Sun G, “An Integrated Model of Infection Risk in a Health-Care Environment," Risk Anal, vol. 26, no. 4, pp. 1085-1094, 2006. [PubMed: 16948699]

[14]. Buttner MP, Cruz P, Stetzenbach LD, and Kilma-Comba AK, "Evaluation of Two Surface Sampling Methods for Detection of Erwinia herbicola on a Variety of Materials by Culture and Quantitative PCR,” Appl, vol. 73, no. 11, pp. 3505-3510, 2007.

[15]. Stuart LC and Jones CW, "Research Article: Re-isolation of bacteria from intentionally contaminated surfaces," BIOS, vol. 77, no. 2, pp. 47-55, 52006.

[16]. Sabbah S, Springthorpe S, and Sattar SA, "A Standard Approach to Assessing Disinfection of Environmental Surfaces: Experiments with a Mixture of Surrogates for Infectious Bioagents," Appl. Environ. Microbiol, Jul. 2010.

[17]. Rabuza U, Šostar-Turk S, and Fijan S, "Efficiency of four sampling methods used to detect two common nosocomial pathogens on textiles," Text. Res. J, vol. 82, no. 20, pp. 2099-2105, Dec. 2012.

[18]. Herzog AB, Pandy AK, Reyes-Gastelum DR, Gerba CP, Rose JB, and Hashsham SA, "Evaluation of Sample Recovery Efficiency for Bacteriophage P22 on Fomites," Appl. Environ. Microbiol, vol. 78, no. 22, pp. 7915-7922, 2012. [PubMed: 22941090]

[19]. Valiante DJ, Schill DP, Bresnitz EA, Burr GA, and Mead KR, "Responding to a Bioterrorist Attack: Environmental Investigation of Anthrax in New Jersey," Appl. Occup. Environ. Hyg, vol. 18, no. 10, pp. 780-785, Oct. 2003. [PubMed: 12959889]

[20]. Silva S, Teixeira P, Oliveira R, and Azeredo J, “Adhesion to and viability of Listeria monocytogenes on food contact surfaces," J. Food Prot, vol. 71, no. 7, pp. 1379-1385, 2008. [PubMed: 18680936]

[21]. Buttner MP, Cruz P, Stetzenbach LD, Kilma-Comba AK, Stevens VL, and Emanuel PA, "Evaluation of the Biological Sampling Kit (BiSKit) for Large-Area Surface Sampling," Appl. Environ. Microbiol, vol. 70, pp. 7040-7045, 2004. [PubMed: 15574898]

[22]. Roman MC and Jan DL, "Proceedings of the NASA Microbiology Workshop," Jun. 2012.

[23]. US EPA, "National Primary Drinking Water Regulations: Long Term 2 Enhanced Surface Water Treatment Rule," Federal Register, Washington DC, USA, 2000.

[24]. Weir MH, Razzollini MTP, Rose JB, and Masago Y, "Water Reclamation Redesign for Reducing Cryptosporidium Risks at a Recreational Spray Park Using Stochastic Models," Water Res., vol. 45, no. 19, pp. 6505-6514, 2011. [PubMed: 22033306]

[25]. Ross SM, Introduction to Probability Models. Academic Press, 2014.

[26]. Weir MH and Haas CN, “A Model for In-vivo Delivered Dose Estimation for Inhaled Bacillus anthracis Spores in Humans with Interspecies Extrapolation,” Environ. Sci. Technoloy, vol. 45, no. 13 , pp. 5828-5833. 
[27]. Masago Y, Shibata T, and Rose JB, "Bacteriophage P22 and Staphylococcus aureus Attenuation on Nonporous Fomites as Determined by Plate Assay and Quantitative PCR," Appl. Environ. Microbiol, vol. 74, no. 18, pp. 5838-5840, 2008. [PubMed: 18621868]

[28]. Rose JB and Haas CN, "A Risk Assessment Framework for the Evaluation of Skin Infections of Antibacterial Soap Washing,” Am. J. Infect. Control, vol. 27, no. 6, pp. S26-S33, 1999. [PubMed: 10586143]

[29]. Kreyszig E, Kreyszig H, and Norminton EJ, Advaned Engieering Mathematics, 10th ed. Wiley, 2011.

[30]. Sheskin DJ, Handbook of Parametric and Nonparametric Statistical Procedures, Fifth Edition 2011.

[31]. Haas CN and Trussell RR, "Frameworks for Assessing Reliability of Multiple Independent Barriers in Potable Water Reuse,” Water Sci. Technol, vol. 38, no. 6, pp. 1-8, 1998. 


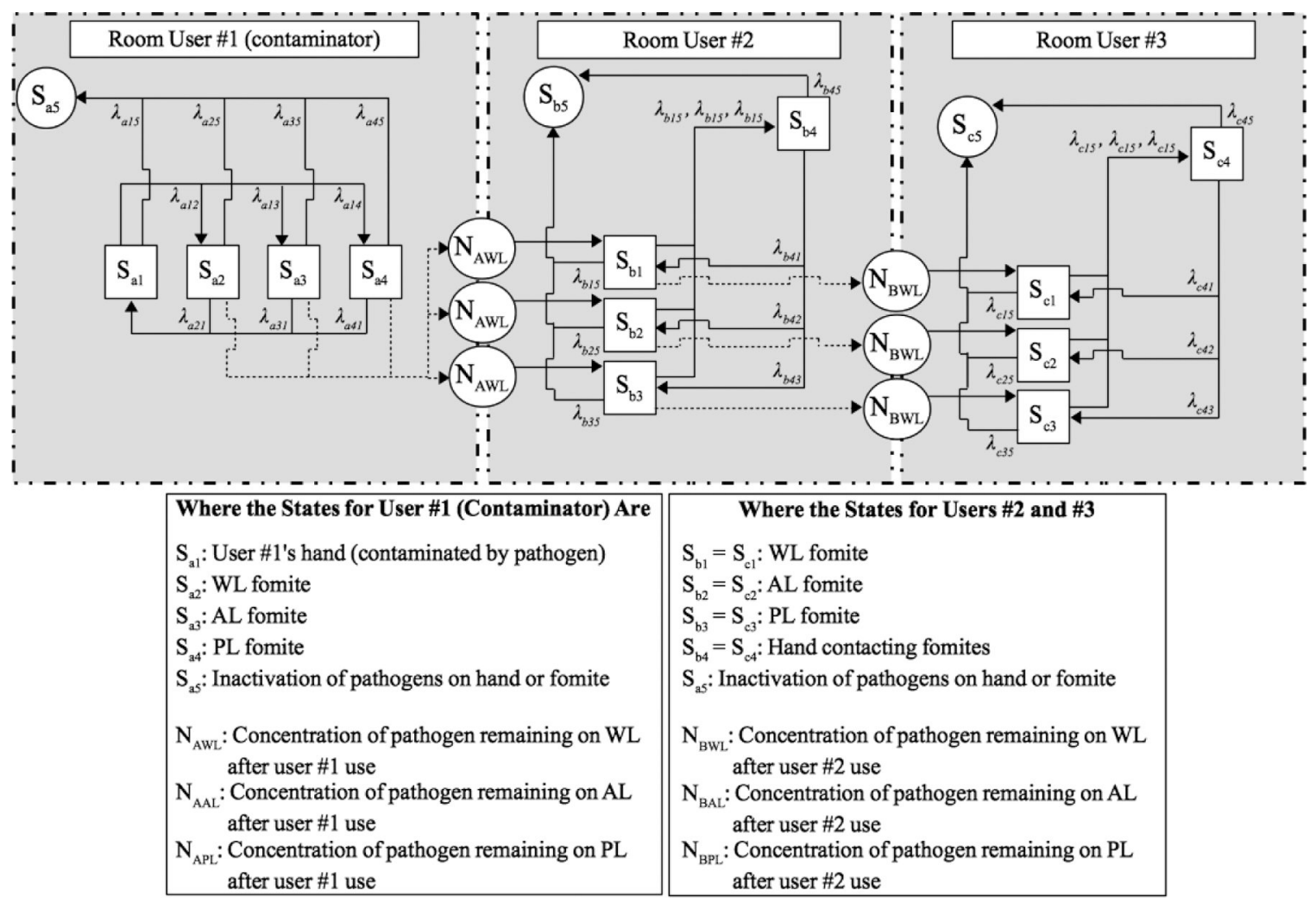

Figure 1.

Conceptual diagram of Markov chain model for fomite contamination and human exposure model. 


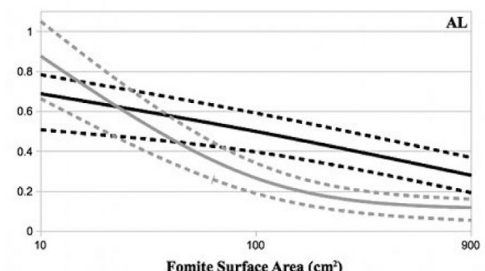

AL

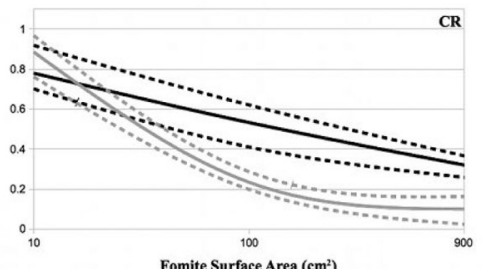

CR
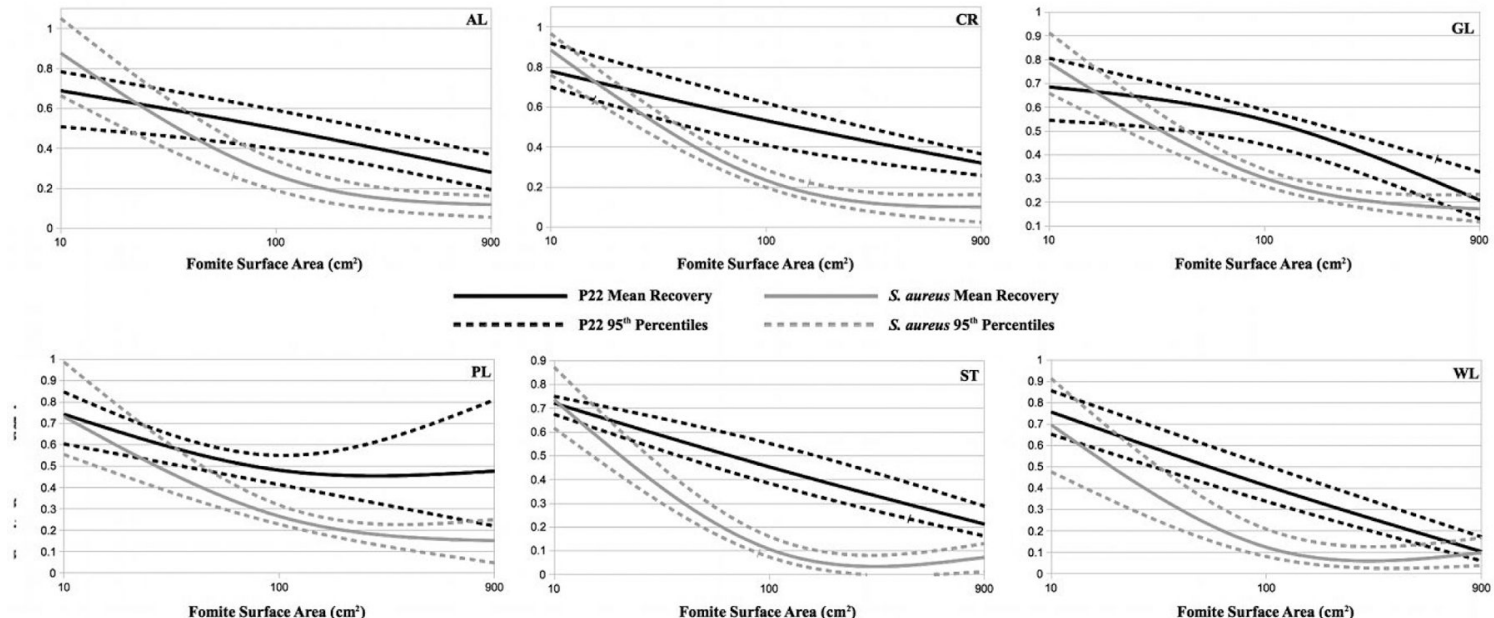

- P22 Mean Recovery
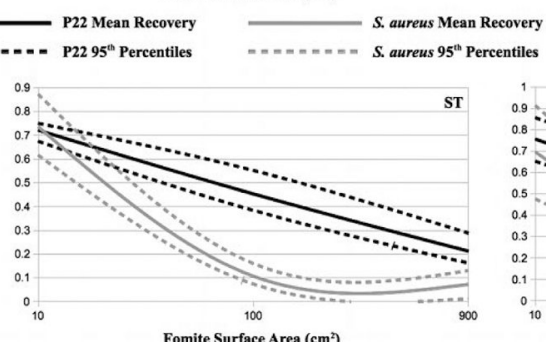

ST

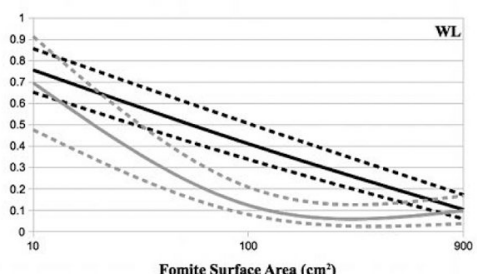

Figure 2.

Recovery efficiencies from the six test fomites, showing median line and one standard deviations in dashed lines. 


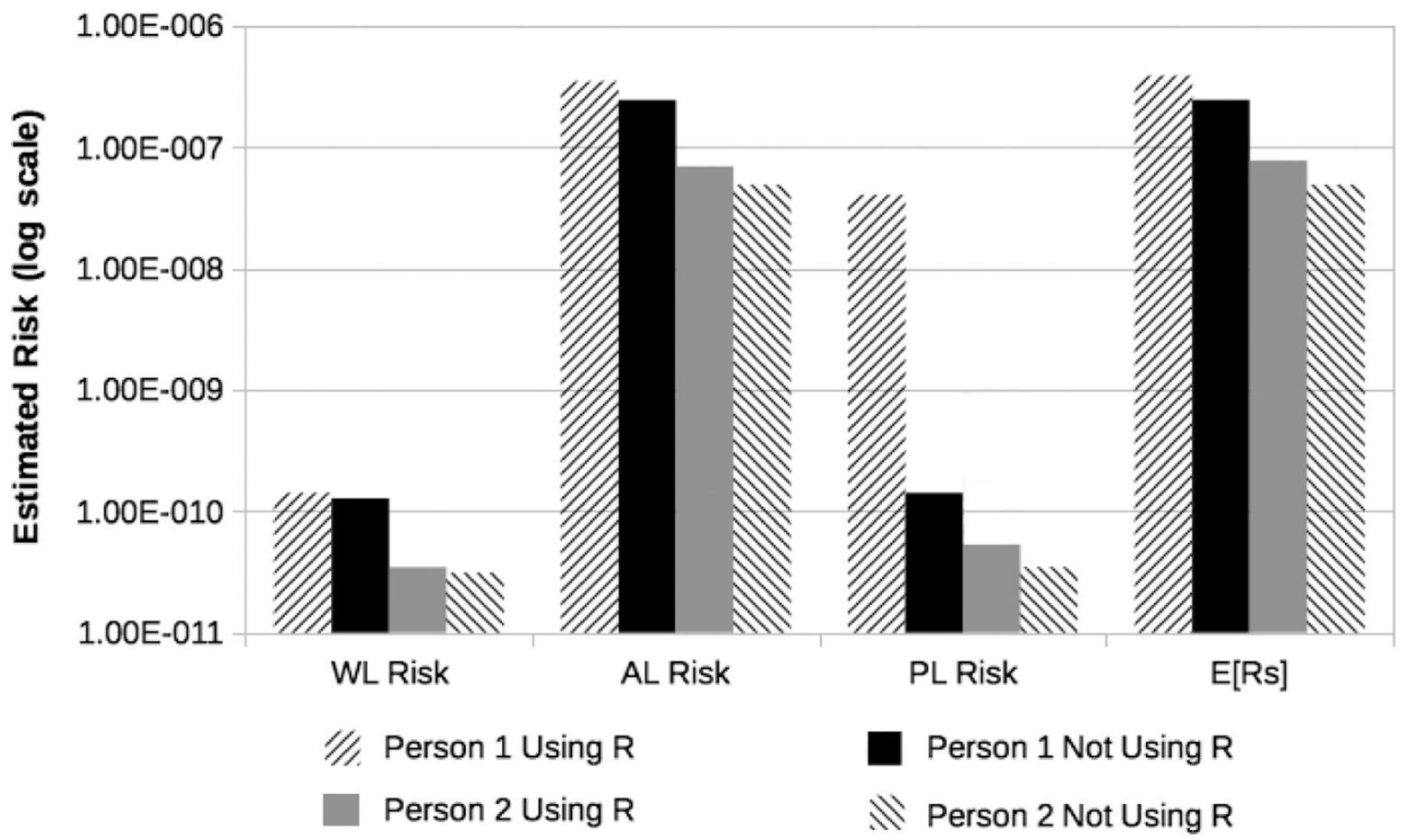

Figure 3.

QMRA model risk results from the test fomites within the exposure time in the QMRA model, $\mathrm{R}$ refers to recovery efficiency. 
- Not Including Recovery Efficiency

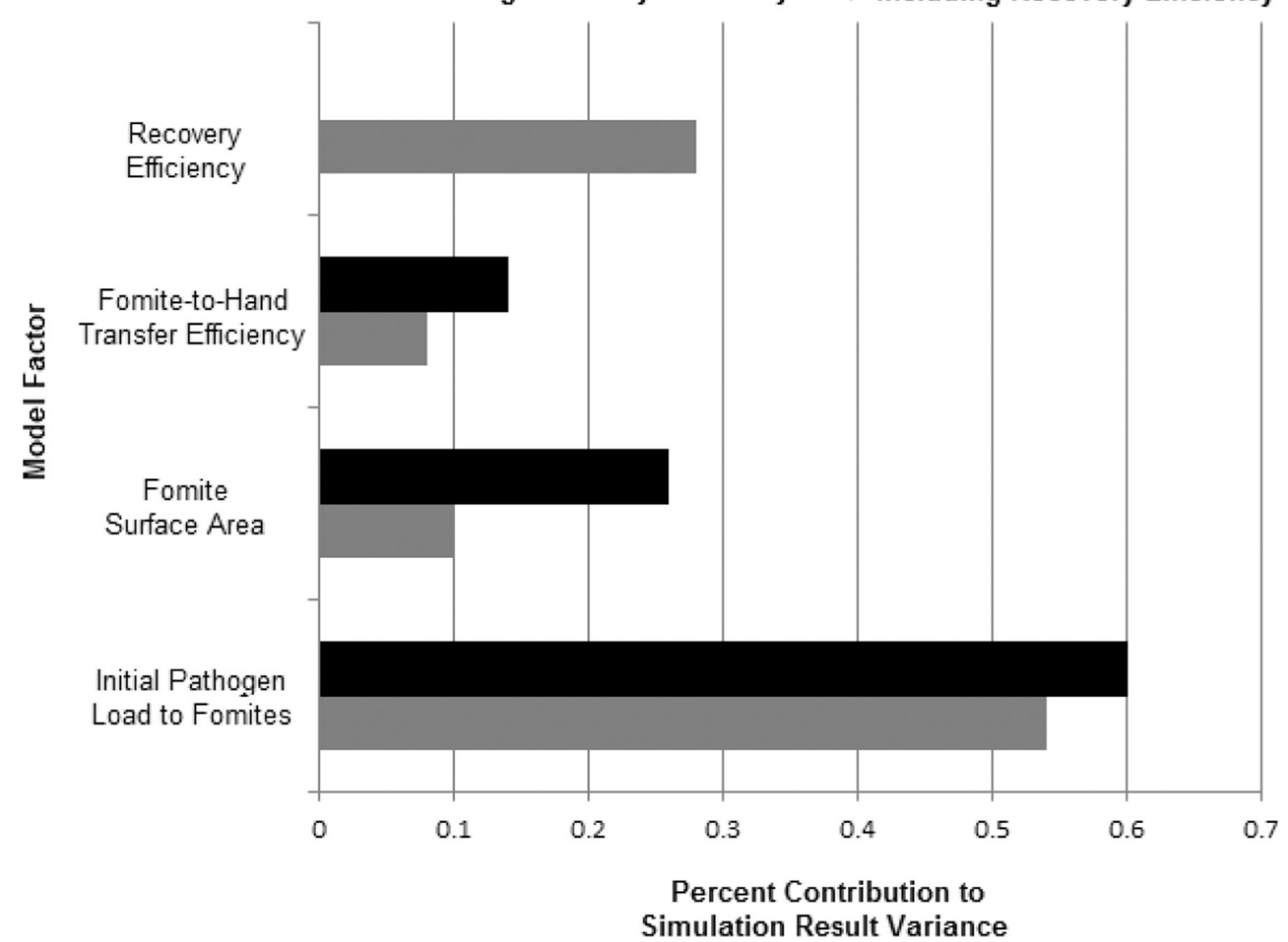

Figure 4.

Sensitivity analysis results from the QMRA model using the differential method. Decay rate and touching frequency had zero effect on the sensitivity since they were constant, therefore not displayed on the figure. 
Table 1.

Evaluation of best recovery tool and eluent for further fomite recovery experiments, values are median percent recoveries plus or minus one standard deviation.

\begin{tabular}{|c|c|c|c|c|c|c|}
\hline \multirow{2}{*}{} & \multicolumn{2}{|l|}{ Recovery Tool } & \multicolumn{3}{l|}{ Recovery Tool Comparison } \\
\cline { 2 - 7 } & Direct Inoculation & \multicolumn{2}{|c|}{ CR Fomite } & \multicolumn{2}{|c|}{ PL Fomite } \\
\cline { 2 - 7 } & Swabs & Wipes & Swabs & Wipes & Swabs & Wipes \\
\hline TSB & $136 \pm 8.9$ & $106 \pm 11$ & $7.8 \pm 1.2$ & $69 \pm 6.0$ & $3.0 \pm 1.3$ & $61 \pm 5.1$ \\
\hline BE & $110 \pm 8.6$ & $94 \pm 8.9$ & $15 \pm 1.6$ & $69 \pm 7.1$ & $13 \pm 1.3$ & $28 \pm 2.2$ \\
\hline PS80 & $114 \pm 7.3$ & $104 \pm 7.1$ & $7.8 \pm 1.0$ & $70 \pm 5.5$ & $22 \pm 3.0$ & $79 \pm 6.6$ \\
\hline Average & $120 \pm 8.3$ & $101 \pm 9.0$ & $10 \pm 1.3$ & $69 \pm 8.9$ & $13 \pm 1.9$ & $56 \pm 4.6$ \\
\hline
\end{tabular}


Table 2.

Effect of initial concentration on the resulting recovery efficiency, values are median percent recoveries plus or minus one standard deviation

\begin{tabular}{|c|c|c|c|c|c|c|}
\hline \multirow{2}{*}{ Fomite } & \multicolumn{3}{|c|}{ P22 Recovery } & \multicolumn{3}{c|}{ S. aureus Recovery } \\
\cline { 2 - 7 } & $\mathbf{1 . 3}\left(\mathbf{1 0}^{\mathbf{2}}\right) \mathbf{p f u} / \mathbf{c m}^{\mathbf{2}}$ & $\mathbf{1 . 6}\left(\mathbf{1 0}^{\mathbf{7}}\right) \mathbf{p f u} / \mathbf{c m}^{\mathbf{2}}$ & Average & $\mathbf{1 . 5}\left(\mathbf{1 0}^{\mathbf{3}}\right) \mathbf{c f u} / \mathbf{c m}^{\mathbf{2}}$ & $\mathbf{3 . 9}\left(\mathbf{1 0}^{\mathbf{6}}\right) \mathbf{c f u}_{\mathbf{c m}} \mathbf{2}$ & Average \\
\hline AL & $68 \pm 6.0$ & $60 \pm 7.5$ & $64 \pm 6.8$ & $87 \pm 14$ & $72 \pm 9.7$ & $79 \pm 12$ \\
\hline CR & $78 \pm 4.3$ & $53 \pm 0.9$ & $66 \pm 2.6$ & $88 \pm 4.0$ & $88 \pm 11$ & $88 \pm 7.5$ \\
\hline GL & $68 \pm 7.2$ & $59 \pm 5.7$ & $63 \pm 6.5$ & $78 \pm 8.1$ & $83 \pm 13$ & $81 \pm 11$ \\
\hline PL & $74 \pm 8.4$ & $65 \pm 13$ & $70 \pm 11$ & $71 \pm 16$ & $89 \pm 14$ & $80 \pm 15$ \\
\hline ST & $72 \pm 1.8$ & $67 \pm 40$ & $69 \pm 21$ & $73 \pm 1.3$ & $65 \pm 37$ & $69 \pm 19$ \\
\hline WL & $76 \pm 7.6$ & $68 \pm 6.2$ & $72 \pm 6.9$ & $68 \pm 5.5$ & $92 \pm 6.8$ & $80 \pm 6.2$ \\
\hline Average & $73 \pm 5.9$ & $62 \pm 12$ & $67 \pm 9.1$ & $77 \pm 8.2$ & $82 \pm 15$ & $80 \pm 6.0$ \\
\hline
\end{tabular}

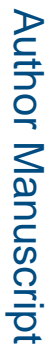

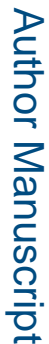

Environ Sci Technol. Author manuscript; available in PMC 2020 May 19. 
Table 3.

Results of recovery experiments for P22 and S. aureus, values are median percent recoveries plus or minus one standard deviation

\begin{tabular}{|c|c|c|c|c|c|c|c|c|}
\hline \multirow{2}{*}{ Fomite } & \multicolumn{5}{|c|}{ P22 Recovery } & \multicolumn{4}{c|}{ S. aureus Recovery } \\
\cline { 2 - 9 } & $\mathbf{1 0 \mathbf { c m } ^ { \mathbf { 2 } }}$ & $\mathbf{1 0 0} \mathbf{c m}^{\mathbf{2}}$ & $\mathbf{9 0 0} \mathbf{c m}^{\mathbf{2}}$ & Average & $\mathbf{1 0 \mathbf { c m } ^ { \mathbf { 2 } }}$ & $\mathbf{1 0 0 \mathbf { c m } ^ { \mathbf { 2 } }}$ & $\mathbf{9 0 0 \mathbf { c m } ^ { \mathbf { 2 } }}$ & Average \\
\hline AL & $68 \pm 6.0$ & $50 \pm 6.0$ & $27 \pm 6.0$ & $49 \pm 6.0$ & $87 \pm 14$ & $26 \pm 2.9$ & $11 \pm 3.0$ & $41 \pm 6.6$ \\
\hline CR & $78 \pm 4.3$ & $54 \pm 5.7$ & $32 \pm 3.9$ & $54 \pm 4.6$ & $88 \pm 4.0$ & $23 \pm 3.0$ & $7.8 \pm 0.2$ & $40 \pm 2.4$ \\
\hline GL & $68 \pm 7.2$ & $55 \pm 4.9$ & $19 \pm 8.6$ & $47 \pm 8.9$ & $78 \pm 8.1$ & $30 \pm 0.6$ & $17 \pm 2.0$ & $42 \pm 3.6$ \\
\hline PL & $74 \pm 8.4$ & $49 \pm 2.5$ & $43 \pm 23$ & $55 \pm 11$ & $71 \pm 16$ & $27 \pm 1.5$ & $13 \pm 8.5$ & $37 \pm 8.7$ \\
\hline ST & $72 \pm 1.8$ & $46 \pm 2.9$ & $21 \pm 3.3$ & $46 \pm 2.7$ & $73 \pm 1.3$ & $10 \pm 3.0$ & $6.6 \pm 4.1$ & $30 \pm 2.8$ \\
\hline WL & $76 \pm 7.6$ & $41 \pm 3.5$ & $10 \pm 4.0$ & $42 \pm 5.0$ & $68 \pm 5.5$ & $12 \pm 4.8$ & $8.4 \pm 4.8$ & $29 \pm 5.0$ \\
\hline Average & $73 \pm 5.9$ & $49 \pm 4.3$ & $25 \pm 8.1$ & $49 \pm 6.1$ & $77 \pm 8.2$ & $21 \pm 2.6$ & $10 \pm 3.8$ & $36 \pm 4.9$ \\
\hline
\end{tabular}

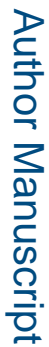

